\title{
Electron Beam Sources using InGaN Semiconductor Photocathodes for Single-shot Imaging Electron Microscope
}

\author{
Tomohiro Nishitani ${ }^{1,2}$, Akihiro Narita ${ }^{3}$, Takeshi Tomita ${ }^{4}$, Shin-ichi Kitamura ${ }^{4}$, Takashi Meguro ${ }^{5}$, \\ Hokuto Iijima $^{5}$, Shingo Fuchi ${ }^{6}$, Masao Tabuchi ${ }^{2}$, Yoshio Honda ${ }^{7}$, Hiroshi Amano ${ }^{7}$ \\ 1. Institute for Advanced Research, Nagoya University, Nagoya, Japan \\ 2. Synchrotron Radiation Research center, Nagoya University, Nagoya, Japan \\ 3. Graduate School of Sciences, The Structural Biology Research Center and Division of Biological \\ Science, Nagoya University, Nagoya, Japan \\ 4. JEOL Ltd., Tokyo, Japan \\ 5. Department of Physics, Faculty of Science Division II, Tokyo University of Science, Tokyo, Japan \\ 6. College of Science and Engineering, Aoyama Gakuin University, Sagamihara-shi, Japan \\ 7. Institute of Materials and Systems for Sustainability, Nagoya University, Nagoya, Japan.
}

Photocathodes using III-V semiconductors with a negative electron affinity (NEA) surface have progressed in high-energy accelerators, and achieved high electron spin polarization [1], large electron beam current [2], small electron energy spread [3]. In addition to the capabilities described above, semiconductor photocathodes have versatile beam performance. The beam current and the emission area are tunable by adjusting the laser power and size, and the beam structure is given a pulse profile by using pulsed laser irradiation. Thus, semiconductor photocathodes provide a quality electron source for not only high-energy accelerators but also industrial applications.

One of the most attractive applications is cryo-electron microscopy (cryo-EM). Currently, the drift of a specimen limits the resolution of cryo-EM images [4], [5]. Exposing the specimen to a high-energy electron beam induces a drift of the sample. Brilot et al. [4] suggested that $0.8 \mathrm{nC}$ is sufficient to provide clear images; however, this caused the drift of $70 \AA$ for $1.25 \mathrm{~s}$ electron beam exposure. To solve this problem, we are working on a new type of cryo-EM using semiconductor photocathodes. If the exposure time exceeds the drift velocity of the sample, and each pulse contains enough charge to provide a clear image, a single-shot imaging electron microscopy will become possible.

The $\mathrm{p}$-InGaN semiconductor was fabricated as a photocathode material for a single-shot imaging electron microscopy [6]. The InGaN photocathode was achieved a quantum efficiency of $4 \%$ at excitation wavelength of $405 \mathrm{~nm}$ around absorption-edge excitation. Furthermore, an pulsed electron beam with a pulse width of $1 \mathrm{~ms}$ and a charge of $12 \mathrm{nC}$ was generated from the InGaN photocathode as shown in Fig. 1.

The semiconductor photocathode gun that size and weight roughly corresponded to those of thermionic cathode gun was also developed, and it was successfully mounted on the conventional $100 \mathrm{kV}$ electron microscope. It contained an NEA surface activation system inside, and had an optical window for irradiating laser to the semconductor photocathode. The maximum acceleration voltage was $50 \mathrm{kV}$, and the maximum extraction current was $10 \mathrm{uA}$. Figure 2 shows a photocathode electron microscope in which the thermionic cathode gun of JEM1200EX was replaced by the photocathode gun. Using the photocathode electron microscope, a grid on a moving specimen holder was imaged by electron beams of direct current and pulse structure respectively. The motion velocity of the specimen holder was two micrometers per second, and the direct current was $20 \mathrm{nA}$, the pulse beam was a peak current of $1.5 \mu \mathrm{A}$, a pulse width of $10 \mathrm{~ms}$ and a repetition frequency of $1 \mathrm{~Hz}$. The beam exposure time for the CCD camera used for these imaging was $500 \mathrm{~ms}$. A blur image was observed with the direct current beam, whereas a clear image was observed with the pulse beam as shown in Fig. 3.

Using the electron detector used by Brilot et al. [4], this technology will improve the throughput of imaging more than 200 times and reduce the sample motion effect. 
References:

[1] T. Nishitani, et al., J. Appl. Phys. 97, 094907 (2005).

[2] G. R. Neil, et al., Phys. Rev. Lett. 84, 662 (2000).

[3] D.A. Orlov, et al., Nucl. Instrum. Methods A 532, 418 (2004).

[4] A. F. Brilot, et al., J. Struct. Biol. 177, 630 (2012).

[5] M. G. Campbell, et al., Structure 20, 1823 (2012).

[6] T. Nishitani, et al., J. Vac. Sci. B 32, 06F901 (2014).

[7] The authors acknowledge funding from SENTAN, JST, Japan.

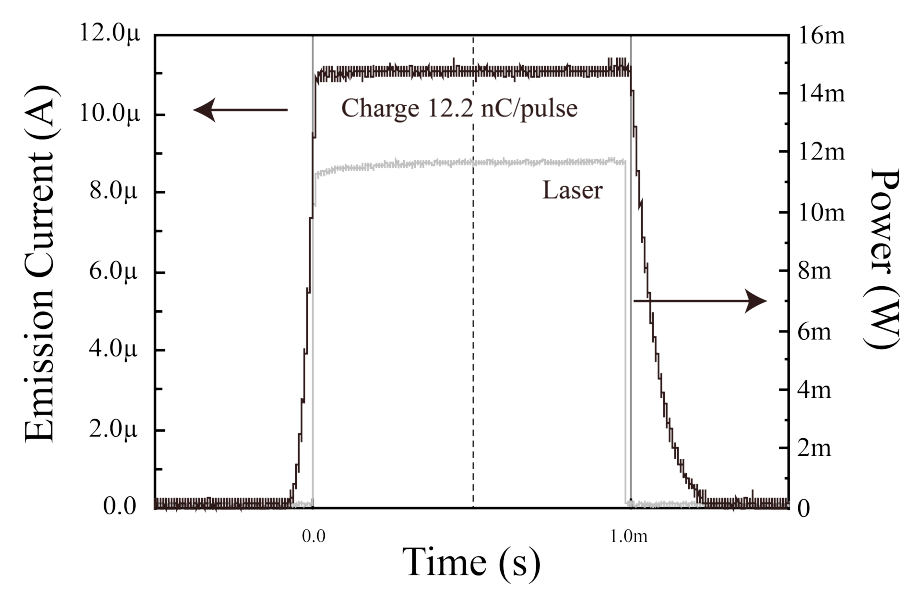

Figure 1. Temporal profiles of pulse electron beam with charge of $12.2 \mathrm{nC}$ for $1 \mathrm{~ms}$ excited from the InGaN photocathode by $12 \mathrm{~mW}$ incident laser power. The time interval is $500 \mathrm{~ms}$, and the excitation wavelength is $406 \mathrm{~nm}$.

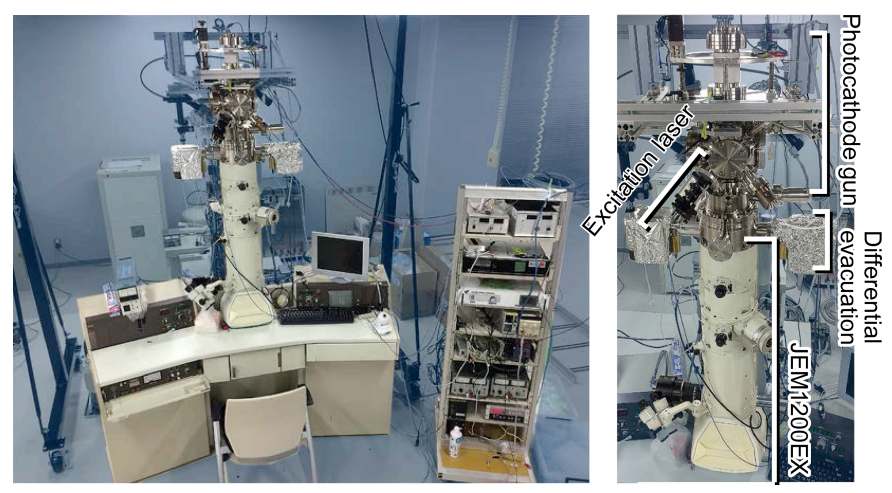

Figure 2. Photocathode electron microscope in which the thermionic cathode gun of JEM1200EX is replaced by the photocathode gun.

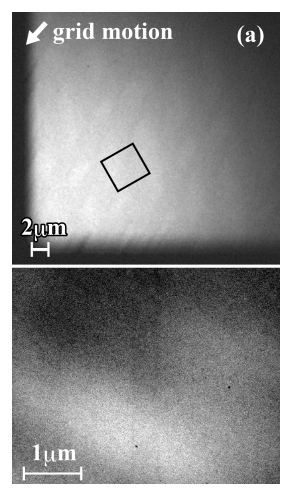

Figure 3.

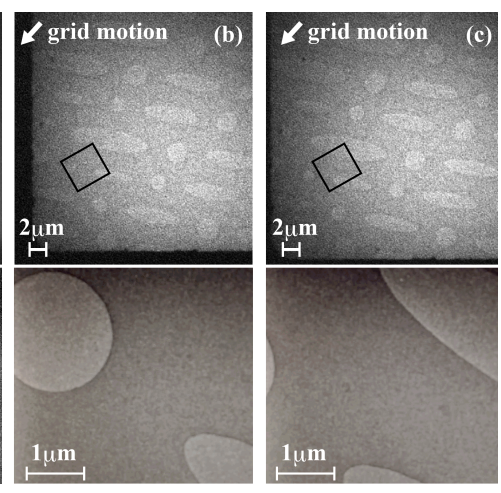

Transmission electron microscopes images of the grid on a moving specimen holder by (a) direct current beam and (b), (c) pulse beam. (c) was the image one second after the image of (b). (a) image was blurred by direct current beam, while (b) and (c) images without blur were observed by pulse beam. 